\title{
COMMENT
}

\section{Addressing rural health disparities in neonatal abstinence syndrome: population-based surveillance and public policy}

\author{
Jean L. Raphael ${ }^{1}$ and Shale L. Wong ${ }^{2}$ on behalf of Pediatric Policy Council \\ Pediatric Research (2019) 85:587-589; https://doi.org/10.1038/s41390-018-0272-9
}

The escalating use and misuse of opioids in the United States has led to a public health crisis with adverse health outcomes, including neonatal abstinence syndrome (NAS). ${ }^{1}$ NAS is a multisystem withdrawal syndrome experienced by infants shortly after birth when antenatal exposure to illegal or prescription drugs is abruptly discontinued at delivery. ${ }^{2}$ Negative sequelae are both short term and long term, including inadequate feeding, disruption of social and behavioral development, and poor school performance. ${ }^{3,4}$ With the rise of the opioid epidemic nationally, rural communities have been especially impacted due to exacerbation of well-documented, deeply rooted health disparities. $^{5,6}$ In this issue, Umer et al. ${ }^{7}$ describe the most current rate of NAS in West Virginia using a statewide surveillance system and evaluate how well the Project WATCH surveillance tool captures NAS data in real time. ${ }^{7}$ West Virginia represents a particularly interesting state to study given that nearly half of its residents live in rural areas. The authors hypothesized that existing studies reliant on administrative data with inherent lag times in availability may underestimate the current opioid crisis. In this study, the authors found that among West Virginia residents, the incidence of NAS was 52.6 per 1000 live births per year, which was much higher than rates reported in previous studies. The authors also found geographic disparities demonstrating some of the highest rates of NAS incidence in rural regions. While the discourse on health disparities has historically focused on race/ ethnicity, there is urgent need to understand and address the root causes of rural health disparities, especially as they pertain to NAS. In this commentary, we focus on disparities experienced by rural populations, the role of surveillance systems in identifying realtime population level disparities, and the implications for public policy in reducing inequities related to NAS.

According the 2010 Census, approximately $20 \%$ of the United States population resides in rural areas, with 47 million adults and 13.4 million children. ${ }^{8}$ Rural populations may experience a number of disparities along the spectrum of access to care, availability of care providers, socioeconomics, and cultural differences, which collectively and adversely impact health outcomes. ${ }^{8}$ In terms of access and availability, rural communities experience a maldistribution of primary care physicians and subspecialists relative to urban communities. Existing workforce shortages are compounded by the fact that near retirement age primary care physicians constitute a large proportion of the rural workforce. ${ }^{5}$ Many rural residents travel longer distances to seek health care and often experience longer wait times for appointments.
Consequently, rural residents may enter care later, with more serious symptoms and advanced medical conditions, requiring more expensive and intensive management relative to their urban peers. ${ }^{6}$ Socioeconomic barriers such as lower family income, higher rates of children living in poverty, and health insurance plans with limited benefits may lead to baseline health disparities and additionally preclude those in rural areas from seeking timely health care.

Cultural factors may also prevent rural residents from seeking appropriate health care services. ${ }^{9}$ Residents may be reluctant to use services due to the lack of anonymity in rural environments of low population density. ${ }^{10}$ Privacy concerns may be especially relevant with respect to opioid addiction and NAS. Those in rural areas may also value self-reliance and individualism and view seeking services more negatively relative to those in urban areas. $^{11}$ The intertwining of these complex and multi-layered disparities have largely contributed to some of the monumental challenges in addressing the opioid epidemic and its downstream sequelae such as NAS. The scope of these disparities has increased the need for real-time, accurate data in order to implement policy change.

With an increasing incidence of NAS and its associated public health burden, population-based surveillance systems have been employed as a central strategy to drive timely policy strategies and prevention initiatives. Health surveillance is the continuous, systematic collection, analysis, and interpretation of health-related data needed for the planning, implementation, and evaluation of public health practice. ${ }^{12}$ In addition to West Virginia, other states (Florida, Georgia, Kentucky, and Tennessee) have implemented surveillance systems or designated NAS as a reportable condition to track NAS rates and inform public health policy. ${ }^{13}$ In 2013, the Tennessee Department of Public Health made NAS reporting mandatory with initiation of a statewide surveillance system. ${ }^{1}$ As with Project WATCH in West Virginia, the Tennessee surveillance system also identified geographic disparities with NAS incidence distribution. Higher rates of NAS were found in Tennessee's eastern, more mountainous counties. The high incidence of NAS in these rural areas was consistent with other indicators of opioid use/misuse in Tennessee, such as opioid prescriptions and overdose deaths.

As clinicians, researchers, and policy makers continue to leverage the promise of population-based surveillance systems to address NAS, they are increasingly identifying a pattern of geographic disparities that demonstrate the vulnerability of rural

${ }^{1}$ Center for Child Health Policy and Advocacy, Baylor College of Medicine, Houston, TX, USA and ${ }^{2}$ Department of Pediatrics, University of Colorado School of Medicine, Aurora, CO, USA

Correspondence: Jean L. Raphael (Raphael@bcm.edu)

Received: 10 December 2018 Accepted: 13 December 2018

Published online: 12 January 2019 
communities. The question for decision makers is how to successfully implement population-based prevention and intervention strategies for NAS without exacerbating underlying disparities experienced by rural communities. Primary prevention strategies to prevent the incidence of NAS include access to preconception care and family planning services and prescription drug monitoring programs (PDMPs). ${ }^{13,14}$ However, underlying inequities experienced by rural communities may render these approaches less effective and exacerbate disparities in outcomes between urban and rural populations. Provider workforce shortages may make access to preconception care and family planning services more difficult. Providers with large patient panels and inability to adopt electronic medical records in rural settings may encounter time constraints and data integration challenges that prevent effective use of PDMPs. In addition to primary prevention strategies, interventions strategies to mitigate the effects of NAS may also be undermined by barriers in rural settings. Such strategies include early identification of infants at risk and standardized treatment of infants with NAS during hospitalization. ${ }^{13}$ However, if families live far away from hospitals, it may be difficult for them to participate in evidence-based activities to mitigate the effects of NAS such as increased family involvement during hospitalization.

Population-based surveillance systems for NAS such as those used by West Virginia and Tennessee have the potential to be a central policy lever in addressing disparities in NAS incidence and outcomes according to geography. First, they can provide comprehensive data on the scope of disparities at small geographic units such as the county level. Second, the findings from these surveillance systems can inform the development of evidence-based NAS interventions tailored towards the specific challenges of high-risk rural communities. Over the past several years, the federal government has implemented legislative initiatives to support surveillance. ${ }^{13}$ The Protecting Our Infants Act of 2015 stipulates that the US Department of Health and Human Services review intra-agency programs related to NAS and prenatal opioid exposure and provide technical assistance to states and American Indian tribes when implementing public health measures, including NAS surveillance systems. The Comprehensive Addiction and Recovery Act of 2016 extends the federal grant program for state-based PDMPs and support for various substance use disorder treatment services for pregnant women and children. In order for the promise of surveillance systems to be fully maximized with respect to reducing rural disparities, further policy change is required in a number of areas. Dedicated grant support through state health departments must be invested in the development of surveillance systems. These grants should include performance benchmarks for addressing rural disparities in prevention, identification, and management of NAS. States with high rates of NAS in rural communities should implement surveillance programs with mandatory NAS reporting. Funds should be specifically allocated to provide administrative and technical support for clinicians in rural communities who may not have the time or resources to collect data or participate in PDMPs. Implementation of standardized NAS care protocols should be promoted in rural community hospitals given similar health outcomes to academic settings when NAS clinical guidelines are used. ${ }^{15}$ Evidence-based strategies to address NAS should be customized to the needs of rural communities. Studies have demonstrated the feasibility and efficiencies gained with telemedicine-based models of care for rural populations. ${ }^{16}$ Policies supporting telemedicine can be used to overcome provider shortages with respect to family planning and preconception care for women who use opioids. Tele-mentoring can additionally be used to help rural clinical providers access experts in opioid addiction and NAS management. ${ }^{17}$ Finally, policy efforts to address the physician workforce shortage, such as increasing rural residency programs, also have the potential to reduce inequities. $^{18}$

NAS has emerged as a significant consequence of the opioid epidemic resulting in long-term detrimental effects on children. With efforts to address NAS within the context of a major public health crisis, decision makers have increasingly relied on the use of surveillance systems as a comprehensive, real-time strategy to inform policy. The availability of real-time data has illuminated substantial inequities experienced in rural communities. Initiatives leveraging surveillance data to tailor policy strategies and interventions may ultimately improve population health while also reducing long-standing rural health disparities.

\section{PEDIATRIC POLICY COUNCIL (PPC)}

Scott C. Denne, MD, Chair, Pediatric Policy Council; Shale L. Wong, MD, MSPH, Representative to the PPC from the Academic Pediatric Association; Jean L. Raphael, MD, MPH, Representative to the PPC from the Academic Pediatric Association; Jonathan Davis, MD, Representative to the PPC from the American Pediatric Society; DeWayne Pursley, MD, MPH, Representative to the PPC from the American Pediatric Society; Scott Rivkees, MD, Representative to the PPC from the Association of Medical School Pediatric Department Chairs; Valerie Opipari, MD, Representative to the PPC from the Association of Medical School Pediatric Department Chairs; Shetal Shah, MD, Representative to the PPC from the Society for Pediatric Research; Joyce Javier, MD, $\mathrm{MPH}, \mathrm{MS}$, Representative to the PPC from the Society for Pediatric Research

\section{ADDITIONAL INFORMATION}

Competing interests: The authors declare no competing interest.

Publisher's note: Springer Nature remains neutral with regard to jurisdictional claims in published maps and institutional affiliations.

\section{REFERENCES}

1. Warren, M. D. et al. Implementation of a statewide surveillance system for neonatal abstinence syndrome-Tennessee, 2013. Morb. Mortal. Wkly. Report. 64, 125-128 (2015).

2. Convertino, I. et al. Neonatal adaptation issues after maternal exposure to prescription drugs: withdrawal syndromes and residual pharmacological effects. Drug Saf. 39, 903-924 (2016).

3. Klinger, G. et al. Long-term outcome following selective serotonin reuptake inhibitor induced neonatal abstinence syndrome. J. Perinatol. 31, 615-620 (2011).

4. Oei, J. L. et al. Neonatal abstinence syndrome and high school performance. Pediatrics 139, 1-10 (2017).

5. Rosenblatt, R. A., Chen, F. M., Lishner, D. M. \& Doescher M. P. The Future of Family Medicine and Implications for Rural Primary Care Physician Supply (WWAMI Rural Health Resource Center, University of Washington, Seattle, WA, 2012).

6. Rost, K., Fortney, J., Fischer, E. \& Smith, J. Use, quality, and outcomes of care for mental health: the rural perspective. Med. Care Res. Rev. 59, 231-265 (2002).

7. Umer A. et al. Capturing the statewide incidence of neonatal abstinence syndrome in real time: the West Virginia experience. Pediatr. Res. PubMed PMID: 30287893 (2018).

8. Basco, W. T. \& Rimsza, M. E., Committee on Pediatric W, American Academy of P. Pediatrician workforce policy statement. Pediatrics 132, 390-397 (2013).

9. Fontanella, C. A. et al. Widening rural-urban disparities in youth suicides, United States, 1996-2010. JAMA Pediatr. 169, 466-473 (2015).

10. Judd, F., Cooper, A. M., Fraser, C. \& Davis, J. Rural suicide-people or place effects? Aust. N. Z. J. Psychiatry 40, 208-216 (2006).

11. Hoyt, D. R., Conger, R. D., Valde, J. G. \& Weihs, K. Psychological distress and help seeking in rural America. Am. J. Community Psychol. 25, 449-470 (1997).

12. Nsubuga, P. et al. in: Disease Control Priorities in Developing Countries (eds Jamison, D. T. et al.) (Washington, DC, Oxford University Press, New York, 2006).

13. Ko, J. Y. et al. CDC grand rounds: public health strategies to prevent neonatal abstinence syndrome. Morb. Mortal. Wkly. Rep. 66, 242-245 (2017).

14. Patrick, S. W., Fry, C. E., Jones, T. F. \& Buntin, M. B. Implementation of prescription drug monitoring programs associated with reductions in opioid-related death rates. Health Aff. 35, 1324-1332 (2016).

15. Friedman, $\mathrm{H}$. et al. Pharmacologic treatment of infants with neonatal abstinence syndrome in community hospitals compared to academic medical centers. J. Perinatol. 38, 1651-1656 (2018). 
Addressing rural health disparities in neonatal abstinence syndrome:... JL Raphael and SL Wong

16. Marcin, J. P., Shaikh, U. \& Steinhorn, R. H. Addressing health disparities in rural communities using telehealth. Pediatr. Res. 79, 169-176 (2016).

17. Komaromy, M. et al. Project ECHO (Extension for Community Healthcare Outcomes): a new model for educating primary care providers about treatment of substance use disorders. Subst. Abus. 37, 20-24 (2016).
18. Department of Health and Human Services Health Resources and Services Administration. Rural Residency Planning and Development Program (Department of Health and Human Services Health Resources and Services Administration). Washington, D.C. 